\title{
Observability of the virialization phase of spheroidal galaxies with radio arrays
}

\author{
Marcella Massardi \\ SISSA/ISAS \\ Via Beirut 2-4, I-34014 Trieste, Italy \\ E-mail: massardiesissa.it \\ Andrea Lapi, Luigi Danese \\ SISSA/ISAS \\ Via Beirut 2-4, I-34014 Trieste, Italy
}

\section{Gianfranco De Zotti}

INAF, Osservatorio Astronomico di Padova

Vicolo dell'Osservatorio 5, I-35122 Padova, Italy

Ronald D. Ekers

Australia Telescope National Facility

CSIRO, P.O. Box 76, Epping, NSW 1710, Australia

\begin{abstract}
In the standard galaxy formation scenario plasma clouds with a high thermal energy content must exist at high redshifts since the proto-galactic gas is shock heated to the virial temperature and extensive cooling, leading to effcient star formation, must await the collapse of massive halos. Massive plasma clouds are potentially observable through the thermal and kinetic Sunyaev-Zel'dovich effects and their free-free emission. We found that the detection of substantial numbers of galaxyscale thermal SZ signals is achievable by blind surveys with next generation radio telescope arrays such as EVLA, ALMA and SKA. This population is even detectable with the $10 \%$ SKA, and wide field of view options at high frequency on any of these arrays would greatly increase survey speed. An analysis of confusion effects and of the contamination by radio and dust emissions shows that the optimal frequencies are those in the range 10-35 GHz.
\end{abstract}

From planets to dark energy: the modern radio universe

October 1-5 2007

University of Manchester, Manchester, UK 


\section{The model and the virial collapse}

We adopt the semi-analytic model laid out in Granato et al. (2004), as updated by Lapi et al. (2006). Briefly, the model assumes that:

- during or soon after the formation of the host dark matter (DM) halo, the baryons falling into the newly created potential well are shock-heated to the virial temperature;

- the hot gas is (moderately) clumpy and cools quickly in the denser central regions, triggering a strong burst of star formation;

- the radiation drag due to starlight acts on the gas clouds, reducing their angular momentum: a fraction of the cool gas falls into a reservoir around the central supermassive black hole (BH), and eventually accretes onto it by viscous dissipation, powering the nuclear activity;

- the energy fed back to the gas by supernova explosions and AGN activity regulates the ongoing star formation and the BH growth. Eventually, the SN and the AGN feedbacks unbind most of the gas from the dark matter potential well, so that the star formation and the $\mathrm{BH}$ activity come to an end on a timescale shorter for the more massive galaxies.

In the redshift range considered here ( $\mathrm{z}>1.5)$, for the massive objects $\left(10^{11.4}<M_{v i r}<10^{13.2} M_{\odot}\right)$ we are dealing with, we have that the only relevant cooling mechanism is free-free emission. We assume that the collapse is spherically symmetric, that all the gas is heated to the virial temperature and that, after virialization, the protogalaxy has a NFW density profile. The model proved to be remarkably successful in accounting for a broad variety of data, including epoch dependent luminosity functions and number counts in different bands of spheroidal galaxies and of AGNs, the local black hole mass function, metal abundances, fundamental plane relations and relationships between the black hole mass and properties of the host galaxies (Lapi et al. 2006).

\section{The signals and the source counts}

In our analysis we considered the free-free emission and the thermal and kinetic SunyaevZel'dovich (SZ) effects from the virialized gas. For the details of the calculations we refer to Massardi et al. (2007). Considering the density distribution, the virial temperature and virial radius of a halo, we can define the flux for each of the signals as functions of the virial mass, redshift and frequency of observation. For an object with $M=10^{12} M_{\odot}$ at $\mathrm{z}=2$ for which the virial temperature is of the order of $T_{v i r} \simeq 1.4 \times 10^{6} \mathrm{~K}$, the mean electron density $n_{e} \simeq 10^{-3} \mathrm{~cm}^{-3}$, the virial radius $r_{v i r} \simeq 10^{6} \mathrm{kpc}$,

$$
\begin{gathered}
S_{\mathrm{ff}}=6.6 \times 10^{-9} \bar{g}_{\mathrm{ff}}\left[T_{\mathrm{vir}}, v(1+z)\right]\left(\frac{1+z}{3}\right)^{7 / 2}\left(\frac{M_{\mathrm{vir}}}{10^{12} M_{\odot}}\right)^{2 / 3} \cdot \\
\cdot\left(\frac{4.8 \times 10^{28} \mathrm{~cm}}{d_{L}}\right)^{2} \exp \left(\frac{-1.9 \times 10^{-6}(v / 20 \mathrm{GHz})}{\left(M_{\mathrm{vir}} / 10^{12} M_{\odot}\right)^{2 / 3}}\right) \mathrm{Jy} \\
S_{\mathrm{tSZ}}=0.6 \times 10^{-7}\left(\frac{1+z}{3}\right)^{5}\left(\frac{M_{\mathrm{vir}}}{10^{12} M_{\odot}}\right)^{5 / 3}\left(\frac{4.8 \times 10^{28} \mathrm{~cm}}{d_{L}}\right)^{2}\left(\frac{g(x)}{0.24}\right) \mathrm{Jy}
\end{gathered}
$$



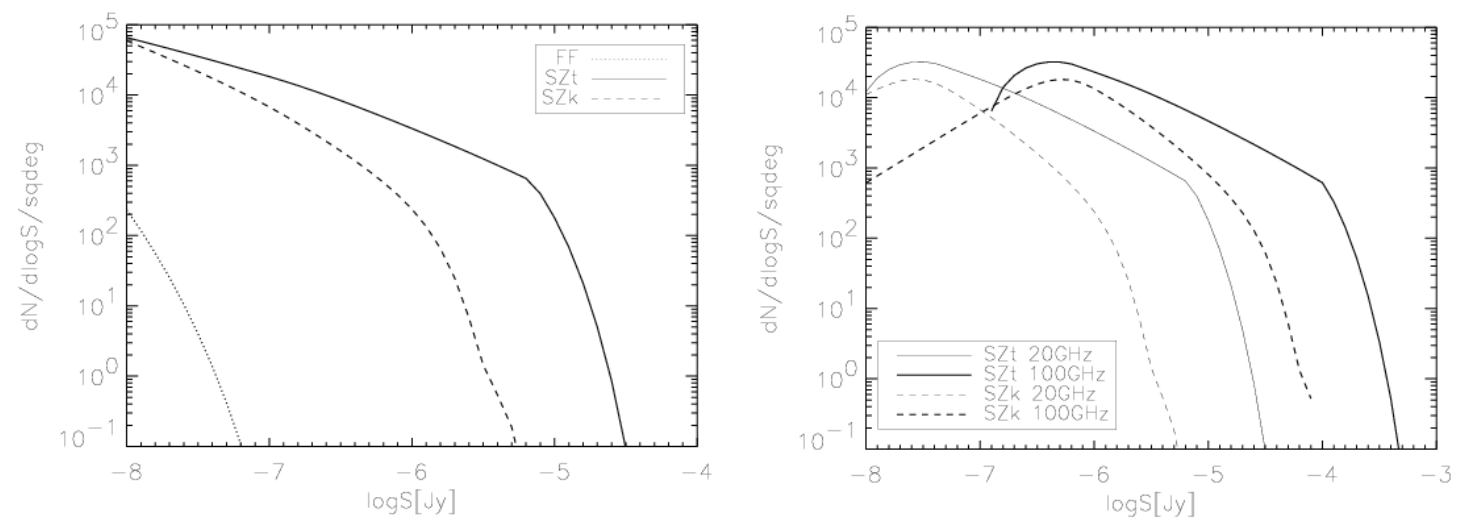

Figure 1: Comparison of the differential source counts (left panel) at $20 \mathrm{GHz}$ of thermal (solid lines) and kinetic (dashed lines) Sunyaev-Zel'dovich effects and free-free (dotted line) and (right panel) comparison of thermal (solid lines) and kinetic (dashed lines) Sunyaev-Zel'dovich effects at $20 \mathrm{GHz}$ and $100 \mathrm{GHz}$ (thin and thick lines respectively). For SZ effects we obviously use the absolute value of the flux. The counts of the kinetic SZ effect include both positive and negative signals. The decline of the counts of SZ effects at the faint end is due to the adopted lower redshift $(z \geq 1.5)$ and halo mass $\left(M_{\mathrm{vir}} \geq 2.5 \times 10^{11} M_{\odot}\right)$ limits.

$S_{\mathrm{kSZ}}=5.3 \times 10^{-8}\left(\left|v_{\text {cloud }}\right| / 393 \mathrm{~km} / \mathrm{s}\right)[(1+z) / 3]^{4}\left(M_{\mathrm{vir}} / 10^{12} M_{\odot}\right)\left(4.8 \times 10^{28} \mathrm{~cm} / d_{L}\right)^{2}[h(x) / 0.12] \mathrm{Jy}$

where $v$ is the frequency, $d_{L}$ is the luminosity distance, $v_{\text {cloud }}$ is the peculiar velocity of the ionized cloud, $g(x)$ and $h(x)$ are the scalings with the adimensional frequency $x=h v / k T$ of the thermal and kinetic SZ fluxes respectively. For such an object the typical angular scale is of 10 arcsec. The mean differential number counts per steradian are given by

$$
\frac{d N(S)}{d \log S}=\int_{\ln \left(z_{\min }\right)}^{\ln \left(z_{\max }\right)} d \ln (z) z \frac{d V}{d z} n[L(S, z), z] \frac{d \log L}{d \log S},
$$

where $d V$ is the comoving volume element. According to our model, for any given $z$ the free-free luminosity of a protospheroid and its thermal SZ signal depend only on its virial mass. The comoving epoch-dependent luminosity function per unit $d \log L, n[L(S, z), z]$, can be computed integrating the formation rate (well approximated by the positive term of the derivative of the Sheth $\&$ Tormen(1999) mass function (Lapi et al. 2006) of virialized objects over the duration of the ionized phase. In the case of the kinetic SZ effect we need also to take into account the redshift dependent distribution of peculiar velocities. The differential source counts at $20 \mathrm{GHz}$ for all the signals, and at 20 and $100 \mathrm{GHz}$ only for the SZ effects are shown in Fig. 1.

\section{Perspective for observation with radio arrays}

In our analysis we considered the telescopes listed in Table 1. The field of view for them corresponds typically the Half Power Beam Width of the antenna: $F O V=1.02 \lambda / D$ where $D$ is the diameter of the antenna dish. Phased array feedhorns add a multiplying factor to this relation, increasing by the same factor the sky area covered in a single pointing. The number of pointings 
Table 1: Main properties of next generation interferometers. The maximum baseline has been calculated considering that the angular size, for the galaxies in the intervals of mass and redshift we are considering, ranges from 5 to 35 arcsec, and requiring a ratio of 5 between amplitude and noise on the visibilities. 10\% SKA has the same properties as SKA, but the number of baselines is $1.25 \times 10^{5}$. SKA parameters refer to the small parabolic dishes version. ATCA values refer to the new $7 \mathrm{~mm}$ broad band system.

\begin{tabular}{lcccc}
\hline & FULL-SKA & ALMA & ATCA & EVLA \\
\hline Frequency $(\mathrm{GHz})$ & $10-20$ & 100 & $35-50$ & 35 \\
Bandwidth(GHz) & 4 & $4 \times 2$ & $2 \times 2$ & 8 \\
Antenna diam. (m) & $12 \mathrm{~m}$ & 12 & 22 & 25 \\
Efficiency & 0.8 & 0.8 & 0.8 & 0.8 \\
$T_{\text {sys }}(\mathrm{K})$ & 50 & 50 & $60-80$ & 75 \\
No. of polariz. & 2 & 2 & 2 & 2 \\
Min. baseline (m) & 15 & 15 & 30.6 & 30 \\
Max. baseline $(\mathrm{km})$ & $1.4-0.7$ & 0.2 & $0.4-0.3$ & 0.4 \\
No. of baselines & $1.25 \times 10^{7}$ & 700 & 10 & 350 \\
\hline
\end{tabular}

necessary to cover a sky area $A_{s}$ is $n_{p}=A_{s} / F O V$. The noise level in an image is given by

$$
\sigma_{\text {image }}=\frac{k_{B} T_{\mathrm{sys}}}{A \eta} \sqrt{\frac{1}{t N_{\mathrm{base}} \Delta v n_{\mathrm{pol}}}}
$$

where $T_{s y s}$ is the system temperature, $A$ is the antenna surface area, $\eta$ is the system (dish and receiver) efficiency, $t$ is the integration time, $N_{\text {base }}$ is the number of baselines short enough to have full sensitivity to observe objects with size between 5 and $35 \operatorname{arcsec}, \Delta v$ is the bandwidth and $n_{p o l}$ is the number of polarizations. If the integral counts of sources scale as $S^{-\beta}$ the number of sources detected in a given area scales as $t^{\beta / 2}$. For a given flux, the number of detections is proportional to the surveyed area, i.e. to $t$. Thus, to maximize the number of detections in a given observing time we need to go deeper if $\beta>2$ and to survey a larger area if $\beta<2$. The number of sources detected above a given flux limit, $S_{\text {lim }}$, within a telescope FOV, $N_{F O V}$, is derived from the source counts and from the above equations, and the results are shown in Fig. 2. The contamination by radio and dust emissions associated to the star formation activity depends on mass and redshift of the objects, but is expected to be stronger than the SZ signal at very low and very high frequencies. In the frequency range $10-35 \mathrm{GHz}$ the thermal SZ is expected to dominate over contaminating signal at least for the most massive objects.

\section{Conclusions}

The detection of substantial numbers of galaxy-scale thermal SZ signals is achievable by blind surveys with next generation radio interferometers. The optimal frequency range for detecting the SZ signal is from 10 to $35 \mathrm{GHz}$, where such signal dominates over the contamination at least for the most massive objects. The high resolution of the SKA will allow us to effectively detect and subtract out confusing sources. Phased arrays at high frequencies could improve the surveying capabilities. 

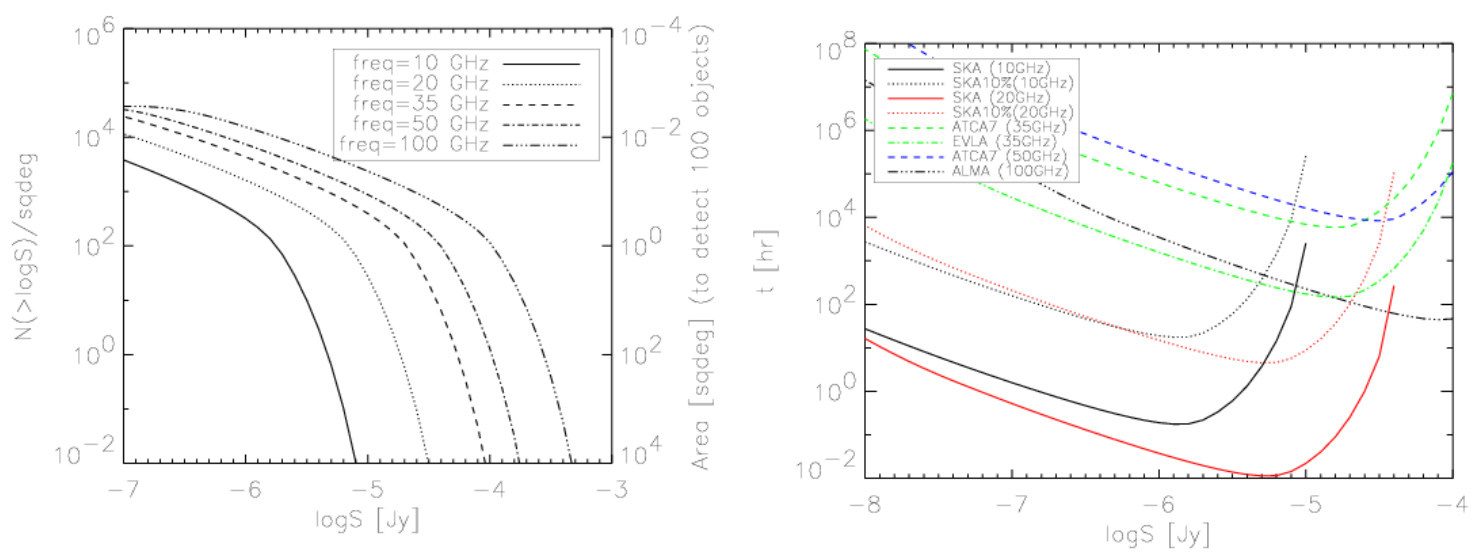

Figure 2: Left Panel. Integral counts and sky area required to detect the thermal SZ effect of 100 protospheroids (right-hand scale) as a function of the absolute value of the "flux" at 10, 20, 35, 50 and $100 \mathrm{GHz}$. Right Panel. On-source time $t_{p} n_{p}$ to detect 100 objects as a function of the thermal SZ limiting flux. The curves have minima at the values of $S_{\text {lim }}$ corresponding to the fastest survey capable of detecting the wanted number of sources. The SKA large effective collecting area allows easily the detection of thermal SZ signals The 10\% SKA requires 100 times more time than the full SKA but is still faster than EVLA or ALMA. If phased array feeds were available at the higher frequencies they would improve these surveying times by a factor of up to 50 .

\section{References}

[1] Granato G. et al., 2004, ApJ, 600, 580

[2] Lapi A. et al., 2006, ApJ, 650, 42

[3] Massardi et al., 2007, MNRAS, Submitted

[4] Sheth R. K., Tormen G., 1999, MNRAS, 308, 119 